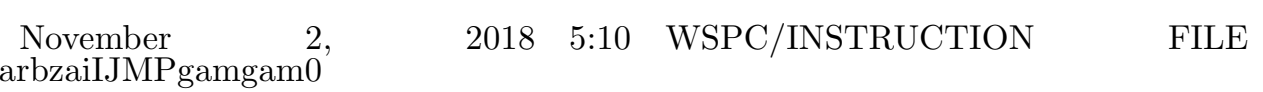

\title{
On a possibility of a consistent interpretation of diboson excesses at LHC
}

\author{
Boris A. Arbuzov \\ M. V. Lomonosov Moscow State University, \\ Leninskie gory 1, 119991 Moscow, Russia \\ arbuzov@theory.sinp.msu.ru \\ Ivan V. Zaitsev \\ M. V. Lomonosov Moscow State University, \\ Leninskie gory 1, 119991 Moscow, Russia \\ zaitsev@theory.sinp.msu.ru \\ Received Day Month Year \\ Revised Day Month Year
}

\begin{abstract}
Recently reported diboson and diphoton excesses at LHC are interpreted to be connected with heavy $W W$ zero spin resonances. The resonances appears due to the wouldbe anomalous triple interaction of the weak bosons, which is defined by well-known coupling constant $\lambda$. The $2 \mathrm{TeV}$ anomaly tentatively corresponds to weak isotopic spin 2 scalar state and the $\gamma \gamma 750 \mathrm{GeV}$ anomaly corresponds to weak isotopic spin 0 pseudoscalar state. We obtain estimates for the effect, which qualitatively agree with ATLAS data. Effects are predicted in a production of $W^{+} W^{-},(Z, \gamma)(Z, \gamma)$ via resonance $X_{P S}$ with $M_{P S} \simeq 750 \mathrm{GeV}$, which could be reliably checked at the upgraded LHC at $\sqrt{s}=13 \mathrm{TeV}$. In the framework of an approach to the spontaneous generation of the triple anomalous interaction its coupling constant is estimated to be $\lambda=-0.02 \pm 0.005$ in an agreement with existing restrictions. Specific predictions of the hypothesis are significant effects in decay channels $X_{P S} \rightarrow \gamma l^{+} l^{-}, X_{P S} \rightarrow l^{+} l^{-} l^{+} l^{-}(l=e, \mu)$.
\end{abstract}

Keywords: anomalous triple boson interaction; W-ball; spontaneous generation of an effective interaction.

PACS numbers: 12.15.Ji; 12.60Cn; 14.70.Fm; 14.70.Hp

\section{Diboson anomalies}

In experiments 1 indications for excesses in the production of boson pairs $W W, W Z, Z Z$ were observed at invariant mass $M_{R} \simeq 2 \mathrm{TeV}$. Data for these processes are also present in works 213. Despite the fact that the wouldbe effect is not finally established yet, the publication causes numerous proposals for an interpretation mostly in terms of theories beyond the Standard Model (see, e.g. 4 ). There are most recent data on $\gamma \gamma$ anomaly at $M(\gamma \gamma) \simeq 750 \mathrm{GeV}$ [5]6, which also caused numerous proposals for an interpretation 7 . As a rule for interpretations of the effects the ad hoc proposals are expressed, each being based on a variant of the Physics beyond the Standard Model. 


$\begin{array}{lrlll}\text { November 2, } & 2018 & 5: 10 & \text { WSPC/INSTRUCTION } & \text { FILE }\end{array}$

However, it seems to be quite advisable to look for options being less radical, which are closely connected with our current knowledge. In this way we are to consider a possibility to explain the anomalies in the framework of non-perturbative contributions in the Standard Model. Then for the heavy resonance 1 the idea naturally appears to prescribe the phenomenon to a resonant state of two $W$-s 8 .

Of course the standard perturbative approach fails for application to such states. However the same assertion is true for usual hadronic states, e.g. light mesons, and here there are non-perturbative approaches, which allows to achieve successes in such problems. As for the light meson physics, we may refer to the well-known nonperturbative effective Nambu - Jona-Lasinio interaction as a tool for an adequate description of the problem. $W W$ bound states and resonances also could appear provided an effective interaction of $W$-s does exist. Such three-boson anomalous effective interaction is not an $a d$ hoc hypothesis. It was proposed a long time ago 9110 and was extensively studied experimentally.

We have studied this option in the framework of a theoretical approach to a possibility of a spontaneous generation of effective interactions 11/12. It is important, that the intensity of the interaction grows with the energy scale upgrowth. The interaction becomes strong enough just on the scale of few $\mathrm{TeV}$, so the appearing of heavy $W W$ resonance becomes natural 8 . Namely, in work $\frac{8}{\text { we have }}$ considered interpretation of the effect in terms of a weak isotopic spin 2 scalar $W W$ state. Indeed, pair of triplets $W^{a}$ could form a resonance state, the so-called $W$-ball. Of course the well known gauge interaction of these bosons with coupling $g\left(M_{W}\right)=0.65$ can not bind them in the resonance state with mass being of a $\mathrm{TeV}$ scale. However, as we already mentioned, provided there exists also the additional effective interaction 910, we come to a formation of a resonance under discussion.

Could this approach also be applied to analogous states? We would consider this problem in the present paper.

\section{A model for the $W W$ resonance}

Now let us consider a possibility of a heavy pseudoscalar resonance in case of an existence of the anomalous three-boson interaction, which in conventional notations $9[10$ looks like

$$
\begin{aligned}
& -\frac{G}{3 !} F \epsilon_{a b c} W_{\mu \nu}^{a} W_{\nu \rho}^{b} W_{\rho \mu}^{c} ; \quad G=-\frac{g \lambda}{M_{W}^{2}} \\
& W_{\mu \nu}^{a}=\partial_{\mu} W_{\nu}^{a}-\partial_{\nu} W_{\mu}^{a}+g \epsilon_{a b c} W_{\mu}^{b} W_{\nu}^{c} ;
\end{aligned}
$$

where $g \simeq 0.65$ is the electro-weak coupling. The best limitations for parameter $\lambda$ read 13

$$
\lambda_{\gamma}=-0.022 \pm 0.019 ; \quad \lambda_{Z}=-0.09 \pm 0.06 ;
$$

where a subscript denote a neutral boson being involved in the experimental definition of $\lambda$. Let us emphasize that $F \equiv F\left(p_{i}\right)$ in definition (1) denotes a form-factor, 
$\begin{array}{lrlll}\text { November } & 2, & 2018 & 5: 10 & \text { WSPC/INSTRUCTION }\end{array}$

arbzaiIJMPgamgam0

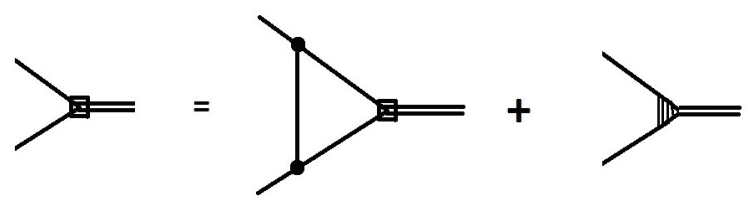

Fig. 1. Diagram form of equation (5). Simple lines represent $W$-s, a double line corresponds to the resonance, black circles correspond to interaction (1), squares correspond to BS wave function. The triangle corresponds to vertex (4).

which is either postulated as in original works $\frac{9 \mid 10}{10}$ or it is just uniquely defined as in works on a spontaneous generation of effective interaction (11) 1112. In any case the form-factor guarantees the effective interaction to act in a limited region of the momentum space. That is it vanishes for momenta exceeding scale $\Lambda_{0}$. Formfactor $F$ is explicitly shown $e . g$. in work 8 . Calculations were done in the framework of an approximate scheme, which accuracy was estimated to be $\simeq(10-15) \% 14$. Wouldbe existence of effective interaction (10) leads to important non-perturbative effects in the electro-weak interaction.

In particular, one might expect resonances to appear in the system of two $W^{a_{-}}$ bosons. A possibility of an appearance of such states (W-balls) was already discussed, e.g. in works $12\left[15\right.$. In the previous work ${ }^{8}$ we have studied the $2000 \mathrm{GeV}$ anomaly and have came to the conclusion, that data may be described in terms of a weak isospin 2 scalar resonance. The effect is due to anomalous interaction (1) and we come to a conclusion 8 , that there is a possibility to describe data ${ }^{1}$ with

$$
\lambda=-0.017 \pm 0.005
$$

Let us turn to recent indications for existence of the other effect: the $\gamma \gamma$ enhancement at invariant mass $M_{\gamma \gamma} \simeq 750 \mathrm{GeV}$. We would consider this effect being explained by existence of zero weak isotopic spin pseudoscalar state $X_{P S}$, which interaction with electroweak bosons is described by the following effective expression

$$
L_{e f f}=\frac{G_{P S}}{4} \delta_{a b} \epsilon^{\mu \nu \rho \sigma} W_{\mu \nu}^{a} W_{\rho \sigma}^{b} X_{P S} ;
$$

Let us consider a Bethe-Salpeter equation for a pseudoscalar resonance consisting of two $W$ corresponding to the weak isospin: $I=0$. With interaction (11) we have the following Bethe-Salpeter equation for state $X_{P S}$ in correspondence to diagrams presented in Fig 1 under assumption of existence of interaction (4).

$$
\begin{aligned}
& \Psi_{P S}=G_{P S}+\frac{G^{2}}{8 \pi^{2}}\left(\frac{1}{6 x} \int_{0}^{x} \Psi_{P S}(y) y^{2} d y-\frac{1}{2} \int_{0}^{x} \Psi_{P S}(y) y d y-\right. \\
& \left.\frac{x}{2} \int_{x}^{\infty} \Psi_{P S}(y) d y+\frac{x^{2}}{6} \int_{x}^{\infty} \frac{\Psi_{P S}(y)}{y} d y\right) ;
\end{aligned}
$$


\begin{tabular}{|lrlll}
$\begin{array}{l}\text { November } \\
\text { arbzailJMPgamgam0 }\end{array}$ & 2018 & $5: 10$ & WSPC/INSTRUCTION & FILE
\end{tabular}

4 B.A. Arbuzov and I. V. Zaitsev

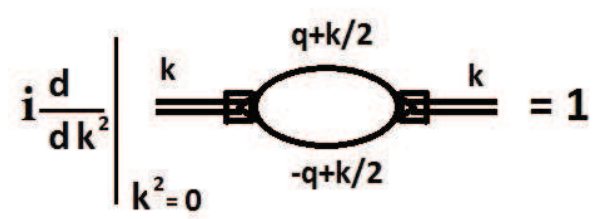

Fig. 2. Diagram representation of normalization condition for coupling $G_{P S}$. Simple lines represent $W$-s, a double line corresponds to the resonance, squares correspond to BS wave function.

where coupling constant $G_{P S}$ is defined by (4). Here in view of large value $M_{X} \simeq$ $0.75 \mathrm{TeV}$ of the wouldbe resonance we neglect $W$ mass. With the following definition of variables

$$
z=\frac{G^{2} x^{2}}{128 \pi^{2}} ; t=\frac{G^{2} y^{2}}{128 \pi^{2}}
$$

we come to the following equation

$$
\begin{aligned}
& \Psi_{P S}(z)=G_{P S}+\frac{4}{3 \sqrt{z}} \int_{0}^{z} \Psi_{R}(t) \sqrt{t} d t-4 \int_{0}^{z} \Psi_{P S}(t) d t- \\
& 4 \sqrt{z} \int_{z}^{\infty} \frac{\Psi_{P S}(t)}{\sqrt{t}} d t+\frac{4 z}{3} \int_{z}^{\infty} \frac{\Psi_{P S}(t)}{t} d t .
\end{aligned}
$$

Equation (7) satisfies condition

$$
\Psi_{P S}(0)=G_{P S} .
$$

By successive differentiations of equation (7) we obtain a Meijer differential equation for function $\Psi_{P S}(z)$

$$
\left[\left(z \frac{d}{d z}+\frac{1}{2}\right)\left(z \frac{d}{d z}\right)\left(z \frac{d}{d z}-\frac{1}{2}\right)\left(z \frac{d}{d z}-1\right)+z\right] \Psi_{P S}(z)=0 .
$$

Then the solution, which fulfill boundary condition both at zero and at the infinity is expressed in terms of Meijer functions (see e.g. $16[17$ ) in the following way

$$
\Psi_{P S}(z)=\frac{G_{P S}}{2} G_{04}^{30}\left(\left.z\right|_{0,1 / 2,1,-1 / 2}\right) .
$$

The normalization condition for Bethe-Salpeter wave function (10) give according to diagram Fig. 2 with account of definition (6) the following relation

$$
\begin{aligned}
& \frac{9}{64 \pi^{2}} \int_{0}^{\infty} d y \Psi_{P S}(y)^{2}=\frac{9 \sqrt{2} G_{P S}^{2}}{16 \pi G} I=1 \\
& I=\int_{0}^{\infty} \frac{G_{04}^{30}\left(\left.t\right|_{0,1 / 2,1,-1 / 2}\right)^{2}}{2 \sqrt{t}} d t=\frac{\pi}{8} .
\end{aligned}
$$

With values $I$ (11), $g=0.65$ and with account of (1) we obtain coupling $G_{P S}$

$$
G_{P S}=\frac{8}{3 M_{W}} \sqrt{g|\lambda| \sqrt{2}}=0.00389 \frac{1}{G e V}
$$


where numerical value corresponds to $\lambda=\lambda_{0}=-0.015$, that is safely inside restrictions (2) and estimates (31). Value (12) corresponds to scale $\Lambda \simeq 0$. We take value (12) for estimates of effects, bearing in mind, that for other values of scale $\Lambda$ coupling $G_{P S}$ is defined by solution (10), namely

$$
G_{P S}(\Lambda)=\frac{G_{P S}}{2} G_{04}^{30}\left(\left.z_{\Lambda}\right|_{0,1 / 2,1,-1 / 2}\right) ; \quad z_{\Lambda}=\frac{G^{2} \Lambda^{4}}{128 \pi^{2}} .
$$

We evaluate the pseudoscalar resonance decay probabilities with $\Lambda_{D}=M_{P S}=$ $750 \mathrm{GeV}$. For estimations of cross sections we take $\Lambda$ in correspondence to maxima of structure functions. That is $\Lambda(\sqrt{s}) \simeq \sqrt{s} / 7$. Then for the decay and for two values $\sqrt{s}=8 \mathrm{TeV}$ and $\sqrt{s}=13 \mathrm{TeV}$ we have the following values for effective coupling $G_{P S}$

$$
\begin{aligned}
& G_{P S}\left(\Lambda_{D}\right)=G_{P S}(0.75 T e V)=0.00362 \\
& G_{P S}(8 T e V / 7)=0.00332 ; \quad G_{P S}(13 T e V / 7)=0.00262 .
\end{aligned}
$$

Thus we have interaction (4) with parameters $G_{P S}$ (12[14) and $M_{P S}=750 \mathrm{GeV}$. We use well-known relation

$$
W^{0}=\cos \theta_{W} Z+\sin \theta_{W} A
$$

and obtain for partial decay widths of the pseudoscalar $X_{P S}$

$$
\begin{aligned}
& \Gamma\left(W^{+} W^{-}\right)=51.3 \mathrm{GeV}(42.9 \%) ; \quad \Gamma(Z Z)=14.8 \mathrm{GeV}(12.4 \%) ; \\
& \Gamma(Z \gamma)=7.8 \mathrm{GeV}(7.6 \%) ; \quad \Gamma(\gamma \gamma)=1.5 \mathrm{GeV}(1.2 \%) ; \\
& \Gamma\left(W^{+} W^{-} Z\right)=35.2 \mathrm{GeV}(29.5 \%) ; \quad \Gamma\left(W^{+} W^{-} \gamma\right)=7.3 \mathrm{GeV}(6.1 \%) ; \\
& \Gamma_{t}\left(X_{P S}\right)=119.4 \mathrm{GeV} .
\end{aligned}
$$

We would present also probabilities for the following specific channels, where $l$ means light lepton $(\mu, e)$

$$
\Gamma\left(l^{+} l^{-} \gamma\right)=0.67 \mathrm{GeV}(0.64 \%) ; \Gamma\left(l^{+} l^{-} l^{+} l^{-}\right)=0.068 \mathrm{GeV}(0.065 \%) .
$$

Then we calculate cross sections for $X_{P S}$ production in $p p$ collisions for $\sqrt{s}=$ $8 \mathrm{TeV}$ and for $\sqrt{s}=13 \mathrm{TeV}$ using values (14). In doing this we use the CompHEP package 18 .

Thus we consider possible pseudoscalar neutral resonance with mass $\approx 750 \mathrm{TeV}$, which mainly decays into

$$
W^{+} W^{-} ; \quad Z Z ; \quad Z \gamma, \quad \gamma \gamma
$$

and also to rare channels (17). According to Table 1 the cross-section of the resonance production at $\sqrt{s}=8 \mathrm{TeV}$ is six times less than at $\sqrt{s}=13 \mathrm{TeV}$. Available $\sqrt{s}=8 \mathrm{TeV}$ data $19|20| 24$ do not contradict to our estimates with account of branching ratios (16). Namely, for $\sqrt{s}=8 \mathrm{TeV}$ we have

$$
\sigma\left(p p \rightarrow X_{P S}\right) \cdot B R\left(X_{P S} \rightarrow \gamma \gamma\right)=292.3 \cdot 0.012=3.51 \mathrm{fb}
$$


\begin{tabular}{|lrlll}
\begin{tabular}{|} 
November \\
arbzaiIJMPgamgam0
\end{tabular} & 2018 & $5: 10$ & WSPC/INSTRUCTION & FILE
\end{tabular}

6 B.A. Arbuzov and I.V. Zaitsev

Table 1

Results for cross-sections of $X_{P S}$ production in $p+p$ collisions at $\sqrt{s}=8 \mathrm{TeV}$ and $\sqrt{s}=13 \mathrm{TeV}$ with $\lambda=-0.015\left(G_{P S}(0)=0.00389 \mathrm{GeV}^{-1}\right)$.

\begin{tabular}{lcc}
\hline channel & $\sigma f b, 8 \mathrm{TeV}$ & $\sigma \mathrm{fb}, 13 \mathrm{TeV}$ \\
\hline$W^{+} X_{P S}$ & 24.1 & 59.7 \\
\hline$W^{-} X_{P S}$ & 6.94 & 20.4 \\
\hline$Z X_{P S}$ & 10.9 & 29.2 \\
\hline$\gamma X_{P S}$ & 3.55 & 9.19 \\
\hline$q(\bar{q})$ jets $X_{P S}$ & 152.3 & 338.8 \\
\hline$W^{+} W^{-} X_{P S}$ & 27.3 & 749.3 \\
\hline$W^{+} Z X_{P S}$ & 51.0 & 537.7 \\
\hline$W^{-} Z X_{P S}$ & 12.3 & 138.2 \\
\hline$W^{+} \gamma X_{P S}$ & 3.03 & 11.5 \\
\hline$W^{-} \gamma X_{P S}$ & 0.78 & 2.86 \\
\hline$\sigma\left(X_{P S}\right)_{t o t}$ & 292.3 & 1896.9 \\
\hline
\end{tabular}

that do not contradict the most recent limitations 21 . Limitations for $W W$ and $Z Z$ decay modes $22 \mid 23$ also do not contradict the results. For example, CMS data 24 give for $750 \mathrm{GeV}$ resonance with width $\simeq 100 \mathrm{GeV}$ limitation $\sigma B R\left(X_{P S} \rightarrow \gamma \gamma\right)<40 \mathrm{fb}$ with prediction (19). Let us note, that our result (17) for channel $X_{P S} \rightarrow l^{+} l^{-} l^{+} l^{-}$ $(l=e, \mu)$ with integral luminosity $L=5.3 \mathrm{fb}^{-1}[19$ gives the following estimate for the number of events

$$
\begin{aligned}
& \sigma\left(X_{P S}, 8 T e V\right) \cdot B R\left(X_{P S} \rightarrow l^{+} l^{-} l^{+} l^{-}\right) \cdot L= \\
& 292.3 \cdot 0.00065 \cdot 5.3=1.01 .
\end{aligned}
$$

It is worth mentioning, that in experimental results at $\sqrt{s}=8 \mathrm{TeV}$ there is one event just at $M\left(l^{+} l^{-} l^{+} l^{-}\right)=750 \mathrm{GeV}$ and no other events for $M\left(l^{+} l^{-} l^{+} l^{-}\right)>$ $600 \mathrm{GeV}$. Of course this coincidence proves nothing due to the poor statistics, we may only state, that results 19 do not contradict our estimates.

Now what for $\sqrt{s}=13 \mathrm{TeV}$ ? First of all let us estimate an effect in channel $\gamma \gamma$. We have for possible number of events with (16) and data from Table 1

$$
N_{\gamma \gamma}=\sigma\left(p p \rightarrow X_{P S}\right) \cdot B R\left(X_{P S} \rightarrow \gamma \gamma\right) \cdot L=22.7 \cdot L\left(f b^{-1}\right) .
$$

Thus we have for $L \simeq 3 \mathrm{fb}^{-1}$ few tens events, that agrees observations $5 \mid 6$.

It may be advisable to study effect not only in channel $X_{P S} \rightarrow \gamma \gamma$ but also in channel $X_{P S} \rightarrow \gamma l^{+} l^{-}$. According to (16) we have

$$
\frac{B R\left(X_{P S} \rightarrow \gamma l^{+} l^{-}\right)}{B R\left(X_{P S} \rightarrow \gamma \gamma\right)}=\frac{0.67}{1.5}=0.45
$$

that is actually only two times smaller than already observed effect in $2 \gamma$. 
$\begin{array}{lllll}\text { November } & 2, & 2018 & 5: 10 & \text { WSPC/INSTRUCTION }\end{array}$

arbzaiIJMPgamgam0

Consistent interpretation of diboson excesses

Let us also calculate the effect for $l^{+} l^{-} l^{+} l^{-}$at $\sqrt{s}=13 \mathrm{GeV}$ in the resonance region $\simeq 750 \mathrm{GeV}$

$$
N\left(l^{+} l^{-} l^{+} l^{-}\right)=\sigma\left(X_{P S}, 13 T e V\right) \cdot B R\left(X_{P S} \rightarrow l^{+} l^{-} l^{+} l^{-}\right) \cdot L=1.23 \cdot L\left(f b^{-1}\right) .
$$

So even for $L \sim 10 \mathrm{fb}^{-1}$ the effect in the four leptons channel may become noticeable. The more so as for this channel background conditions are favorable 19 . Effects $X_{P S} \rightarrow \gamma l^{+} l^{-} ; X_{P S} \rightarrow l^{+} l^{-} l^{+} l^{-}$with intensities (22, 23) would confirm definitely the interpretation of the $750 \mathrm{GeV}$ state being W-ball. Note, that existing limitations on a possible extra contribution of decay $X_{P S} \rightarrow \gamma Z$ with invisible decay

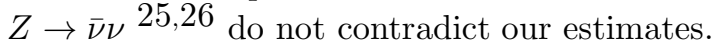

Let us remind, that all the estimates were made with $\lambda=-0.015$. Calculations for another value of $\lambda$ are straightforward with prescriptions of the present work.

\section{Conclusion}

Existence of W-balls would testify for anomalous gauge interaction (11), which would be due to non-perturbative effects in the electroweak interaction. Thus we could come to important conclusion, that non-perturbative contributions are appropriate not only to QCD, but to the electroweak interaction as well. In this case the anomalies in the electroweak boson pair production do not contradict the Standard Model and do not need extra efforts for a choice of a theory beyond the SM.

Data on effects under discussion might give information on a value of parameter $\lambda$. According to our considerations it could be expected in range $\lambda=$ $-0.015 \pm 0.005$. Of course experiments on direct measurement of $\lambda, e . g$. in processes of $W^{+} W^{-}, W^{ \pm} Z(\gamma)$ production are also quite desirable.

We would emphasize, that in case of a success, the wouldbe fact of a simultaneous matching of two difficult for explanation effects in the framework of our approach would be quite instructive. It might serve as a confirmation of non-perturbative method 1112 in case of realization of the predictions being discussed here. This approach could serve for achieving of the additional information on links between fundamental parameters of the Standard Model (see e.g. 12 ).

\section{Acknowledgments}

The work is supported in part by the Russian Ministry of Education and Science under grant NSh-3042.2014.2.

\section{References}

1. G. Aad et.al. (ATLAS Collaboration), J. High Energy Phys., 1512 (2015) 055.

2. V. Khachatryan et.al. (CMS Collaboration), J. High Energy Phys., 1408 (2014) 173.

3. V. Khachatryan et.al. (CMS Collaboration), J High Energy Phys., 1408 (2014) 174.

4. D. Gonsalves, F. Krauss and M. Spannovsky, Phys. Rev. D92 (2015): 053010.

5. ATLAS collaboration, Search for resonances decaying to photon pairs in 3.2 $\mathrm{fb}^{-1}$ of pp collisions at $\sqrt{s}=13 \mathrm{TeV}$ with the ATLAS detector, Tech. Rep. ATLAS-CONF2015-081, CERN, Geneva, 2015. 
$\begin{array}{lrlll}\text { November } 2, & 2018 & 5: 10 & \text { WSPC/INSTRUCTION } & \text { FILE }\end{array}$

arbzaiIJMPgamgam0

8 B.A. Arbuzov and I.V. Zaitsev

6. CMS collaboration, Search for new physics in high mass diphoton events in protonproton collisions at $\sqrt{s}=13 \mathrm{TeV}$, Tech. Rep.CMS-PAS-EXO-15-004, CERN, Geneva, 2015 .

7. J. Ellis et al., J. High Energy Phys., 1603 (2016) 180.

8. B. A. Arbuzov and I. V. Zaitsev, Int. J. Mod. Phys. A30 (2015): 1550221.

9. K. Hagiwara, R.D. Peccei, D. Zeppenfeld and K. Hikasa, Nucl. Phys. B282 (1987) 253.

10. K. Hagiwara, S. Ishihara, R. Szalapski and D. Zeppenfeld, Phys. Rev. D48 (1993) 2182.

11. B. A. Arbuzov, Eur. Phys. J. C61 (2009) 51.

12. B. A. Arbuzov and I. V. Zaitsev, Phys. Rev. D85 (2012): 093001.

13. K. A. Olive et al. (Particle Data Group), Review of particle physics, Chin. Phys. C38 (2014): 090001.

14. B. A. Arbuzov, Theor. Math. Phys. 140 (2004) 1205.

15. B. A. Arbuzov and I. V. Zaitsev, Int. J. Mod. Phys. A27 (2012): 1250012.

16. H. Bateman and A. Erdélyi, Higher Transcendental Functions V.1, McGraw-Hill, NewYork, Toronto, London, 1953.

17. B. A. Arbuzov, Non-perturbative Effective Interactions in the Standard Model, De Gruyter, Berlin, 2014.

18. E. E. Boos et al. (CompHEP Collaboration), Nucl. Instr. Meth. A534 (2004) 250.

19. V. Khachatryan et al. (CMS Collaboration), Phys. Lett. B721 (2013) 190.

20. G. Aad et al. (ATLAS Collaboration), Phys. Rev. Lett 113 (2015): 71801.

21. G. Aad et al. (ATLAS Collaboration), Phys. Rev. D92 (2015): 032004.

22. G. Aad et al. (ATLAS Collaboration), Eur. Phys. J. C75 (2015) 209.

23. G. Aad et al. (ATLAS Collaboration), Phys. Lett. B755 (2016) 285.

24. V. Khachatryan et al. (CMS Collaboration), Phys. Lett. B750 (2015) 494.

25. V. Khachatryan et al. (CMS Collaboration), Phys. Lett. B755 (2016) 102.

26. G. Aad et al. (ATLAS Collaboration), arXiv: 1604.01306 [hep-ex] (2016). 